\title{
A Review Of Going Concern Prediction Studies: 1976 To Present
}

Jodi L. Bellovary, (Email: jodibellovary@charter.net), Marquette University Don E. Giacomino, (Email: Don.Giacomino@marquette.edu), Marquette University Michael D. Akers, (Email: Michael.Akers@marquette.edu), Marquette University

\begin{abstract}
In 1962, the Securities and Exchange Commission (SEC) was the first to address going concern issues with Accounting Series Release (ASR) No. 90. Then, in 1963, the AICPA issued Statement on Auditing Procedures (SAP) No. 33, in response to ASR No. 90. Both ASR No. 90 and SAP No. 33 addressed qualifications for issues that were unresolved and the results of which were indeterminable at the statement date. Soon after the issuance of Statement on Auditing Standards (SAS) No. 2 in 1974, researchers began to conduct studies on going concern issues. This paper provides a comprehensive review of the literature on going concern studies and updates studies by Mutchler (1983) and Asare (1990) which provide detailed reviews of the evolution of the going concern report and requirements of the standards related to auditors' assessment of going concern. Since SAS No. 2, the profession has not provided additional guidance on going concern. Even the Sarbanes-Oxley Act of 2002 (SOX), makes no modifications to the requirements for considering going concern and the Public Company Accounting Oversight Board has not issued guidance addressing going concern. Starting with the first going concern prediction study [McKee, 1976], this paper identifies 27 models developed for predicting the going concern opinion and identifies the primary methods used for model development; multivariate discriminant analysis (MDA), logit analysis, probit analysis, and neural networks are. This paper also identifies; the most popular type of focused model and identifies three non-U.S. firm models, the number of factors considered in any one study, and the predictive abilities of the models. The paper also provides an annotated bibliography for the 27 models.
\end{abstract}

\section{INTRODUCTION}

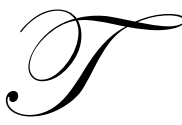

he literature on going concern prediction dates back to 1976, shortly after the issuance of Statement of Auditing Standards (SAS) No. 2, which was the first SAS to detail specific considerations for the auditor's assessment of a firm's going concern status. Since then, several different types of models have been developed for predicting the going concern opinion. The models vary by the types of firms for which the models were developed, how many and which factors are considered, and the methods used to develop the models. For example, Kida [1980] developed a five-factor multivariate discriminant analysis model for manufacturing firms, while Cormier et al. [1995] presented a sixteen-factor logit model for Canadian firms.

This paper continues with a discussion of the auditing standards related to going concern. Next, the authors provide a summary of the literature on going concern prediction. Models are compared based on purpose, development method, and factors/variables. The fourth section analyzes the results of going concern prediction studies. The next section provides conclusions and suggestions for future research. Last, the authors provide an annotated bibliography for going-concern studies since 1976. 


\section{AUDITING STANDARDS RELATED TO GOING CONCERN}

Prior to 1962, there was no formal professional guidance regarding the assessment of a firm's going concern status. ${ }^{1}$ Then, in 1962, the Securities and Exchange Commission (SEC) was the first to address going concern issues when the Commission issued Accounting Series Release (ASR) No. 90. In 1963, the AICPA spoke to the going concern issue with Statement on Auditing Procedures (SAP) No. 33, in response to ASR No. 90. Both ASR No. 90 and SAP No. 33 addressed qualifications for issues that were unresolved and the results of which were indeterminable at the statement date.

In 1974, the AICPA issued SAS No. 2, which provided the first specific discussion of items that were important to consider when assessing a firm's going concern status. With the subsequent issuance of SAS No. 34 in 1981 and SAS No. 59 in 1988, the AICPA further addressed this topic. The three primary changes from SAS No. 34 to SAS No. 59 were the requirements that: (1) auditors consider the client's going concern status for every audit engagement, (2) the audit report be modified if there is substantial doubt about the entity's going concern status, and (3) the audit report include an explanatory paragraph regarding the substantial doubt [Asare, 1990].

For the past few decades, the going concern opinion has been a topic of debate. In 1978, the Commission on Auditors' Responsibilities (Cohen Commission) provided recommendations for improving and better defining the responsibilities of independent auditors. With regard to going concern uncertainties, the Cohen Commission suggested:

If uncertainty about a company's ability to continue operations is adequately disclosed in its financial statements, the auditor should not be required to call attention to that uncertainty in his report... If the auditor does not believe disclosure is sufficient to portray the company's financial position, he should modify his opinion because the financial statements do not present the company's financial position in conformity with generally accepted accounting principles (Cohen Commission 1978, 30).

Several studies have considered, with mixed results, the usefulness of the going concern opinion (see Asare [1990] for a discussion of these studies).

Despite the recent high-profile cases of accounting fraud and the subsequent changes in the profession, no further guidance has been issued on going concern. The Sarbanes-Oxley Act of 2002 (SOX), issued in the wake of the recent accounting frauds, makes no modifications to the requirements for considering going concern. To date, the Public Company Accounting Oversight Board, created by SOX to oversee audits of public companies, has not issued guidance addressing going concern. Thus, SAS No. 59 still remains the current authoritative guidance available to auditors regarding the going concern status of a firm.

\section{SUMMARY OF GOING CONCERN PREDICTION STUDIES}

The appearance of going concern studies in the research literature coincides with the issuance of standards addressing going concern. The first going concern prediction study [McKee, 1976] was published shortly after the issuance of SAS No. 2 in 1974. The next few studies ([Kida, 1980]; [Williams, 1982]; [Mutchler, 1985]) were published around the issuance of SAS No. 34 in 1981. Again, we see a number of studies published just before and within a few years after the issuance of SAS No. 59 in 1988. Going concern prediction studies are also impacted by events in the professional and business community. For example, Geiger and Raghunandan [2002] examined the change in the rate of going concern reports issued before and after the Private Securities Litigation Reform Act of 1995. Similarly, Geiger et al. [2005] investigated the propensity of auditors to issue going concern opinions before and after the recent high-profile accounting cases and the issuance of SOX.

\footnotetext{
${ }^{1}$ See Mutchler [1983] and Asare [1990] for detailed reviews of the evolution of the going concern report and the requirements of standards related to auditors' assessments of going concern.
} 
There is often an overlap in the literature of bankruptcy and going concern topics. Those two topics are tied together by the fact that a firm's going concern status may be questionable prior to filing bankruptcy. Under current authoritative guidance, when going concern problems become apparent, the auditor clearly should issue a going concern report to a firm . Further, going concern prediction studies often use samples of bankrupt firms versus nonbankrupt firms to assess model accuracy. Table 1 lists 27 models that have been developed for predicting the going concern opinion of a firm. These models were developed specifically for predicting the going concern opinion that should be issued to a firm, not for predicting whether a firm will go bankrupt.

The models are listed in Table 1 first by year of publication, then alphabetically within the year. During the 1970's, there was only one going concern model [McKee, 1976] published . However, the number of models increased to seven in the 1980's and sixteen in the 1990's, and the early part of this decade has seen three models published (2000 to 2003). Table 1 includes the purpose of the model, type of model, number of factors, and model accuracy. When more than one method was used to develop models within a study, the study is listed only once in the table with the results for the primary methods used in the study. For example, Harris [1989] used a recursive partitioning algorithm, multivariate discriminant analysis, and logit analysis to develop models. Therefore, the study is listed once in the table with the results for all three methods.

\section{Focused Versus Unfocused Models}

As indicated in footnote 1 of the table, unless otherwise specified, the models are assumed to have been developed for application to medium-to-large manufacturing and retail firms (SIC codes 2000 to 3999 and 5000 to 5999). If a model is more narrowly focused, it is indicated in italics in the "purpose of model" column. The most popular type of "focused" model is for manufacturing firms. These five models are: Kida [1980]; Williams [1982]; Mutchler [1985]; Hansen et al. [1992]; Klersey and Dugan [1995]. Two models ([Dopuch et al., 1987]; [Menon and Schwartz, 1987]) were developed for application to any group of firms with SIC codes other than 6000-6999 (financial, insurance, and real estate firms). One model [Casterella et al., 2000] was developed for application to firms with SIC codes below 6000. The trend seems to be moving away from the development of focused models as most of the focused models were developed in 1995 and earlier.

\section{Global Studies}

While the ability of firms to continue in business is a concern for firms in any country, most studies have developed going concern prediction models for U.S. firms. However, there are a small number of studies that have developed models for non-U.S. firms. These include models for firms in:

- $\quad$ Belgium [Gaeremynck and Willekens, 2003]

- Canada [Cormier et al., 1995]

- UK [Barnes and Huan, 1993]

The paucity of international studies on going concern prediction suggests that the going concern issue is of greater importance in the U. S. than in the rest of the world.

\section{Model Types}

Multivariate discriminant analysis (MDA), logit analysis, probit analysis, and neural networks are the primary methods that have been used for model development. ${ }^{2}$ MDA classifies firms into groups (non-going concern or going concern) based on each firm's characteristics (ratios/factors). Based on sample observations, coefficients are calculated for each characteristic (ratio). The products of the ratios and their coefficients are summed to give a discriminant score, allowing classification of the firm. Logit analysis and probit analysis take into account the probability that the firm will be classified as a going concern or non-going concern. The main difference between these two methods is that probit analysis requires non-linear estimation [Dimitras et al., 1996]. There are several

\footnotetext{
${ }^{2}$ See Dimitras et al. [1996] for more detailed descriptions of the various methods.
} 
different types of neural network methods; however, the details of these methods are beyond the scope of this paper. Basically, neural networks analyze inputs to find patterns and develop a model capable of a decision-making process. Several sample cases are run during the "training" mode, during which the network "learns" the decision-making process. The "testing" mode is used to validate the neural network model using hold-out sample data.

Table 1 breaks down the primary methods for model development used in the studies listed in broken down by time period as summarized below:

\begin{tabular}{cccccc} 
& $\begin{array}{c}\text { Discriminant } \\
\text { Analysis }\end{array}$ & $\begin{array}{c}\text { Logit } \\
\text { Analysis }\end{array}$ & $\begin{array}{c}\text { Probit } \\
\text { Analysis }\end{array}$ & $\begin{array}{c}\text { Neural } \\
\text { Networks }\end{array}$ & Other \\
\hline 1970's & 1 & & & & \\
1980's & 3 & 2 & 2 & 7 & 4 \\
1990's & 1 & 9 & 2 & & 1 \\
2000's & & 2 & & $\mathbf{7}$ & $\mathbf{7}$ \\
Overall & $\mathbf{5}$ & $\mathbf{1 3}$ & $\mathbf{4}$ & \\
\hline
\end{tabular}

[Note: Six studies had more than one method which could be considered "primary"; therefore, the number of total studies listed exceeds 27. "Other" methods include, for example, judgmental and recursive partitioning algorithms.]

Logit analysis has been used most often for developing going concern prediction models, followed in popularity by neural networks. There does not appear to be a pattern to the type of method used for model development. For example, the primary methods used for bankruptcy prediction model development shifted over time from MDA to logit and probit analysis to neural networks [Bellovary et al., 2005]. We do not see a discernible trend for going concern studies.

\section{Model Factors (Variables)}

The number of factors considered in any one study ranges from five to 117 . A total of 237 different factors are used in the studies. Two hundred twelve (212) of the factors are utilized in only one or two of the studies. Table 2 lists the sixteen factors that are considered in five or more of the studies. The factor most common to multiple studies is the ratio of Current Assets to Current Liabilities (Current Ratio), included in fourteen studies. The second most common factor is the ratio of Net Income to Total Assets (Return on Assets), considered in eleven studies.

The number of factors considered in studies broken down by time period is:

\begin{tabular}{lccc} 
& Minimum & Maximum & Average (Rounded) \\
\hline 1970's & 7 & 7 & 7 \\
1980's & 5 & 25 & 11 \\
1990's & 5 & 117 & 16 \\
2000's & 8 & 12 & 10 \\
Overall & $\mathbf{5}$ & $\mathbf{1 1 7}$ & $\mathbf{1 4}$ \\
\hline
\end{tabular}

There has been some fluctuation in the range of the number of factors used in studies; however, the average has remained fairly constant around ten to eleven factors. 


\section{Validation Methods}

Jones [1987] pointed out the need for an appropriate validation method when developing and testing models and suggested the use of a hold-out sample to test external validity. Many studies use the Lachenbruch (or "jackknife") method where one observation is withheld from the estimation sample and its classification predicted. This process is repeated until each observation has been withheld and predicted. Where the sample size is small, the Lachenbruch method is acceptable and often required. However, a better indication of validity is obtained through the use of a hold-out sample (a separate set of observations). Applying the model to the new set of observations one is able to acquire a stronger measure of the model's predictive accuracy. The "model accuracy" column of Table 1 indicates whether the results presented are based on tests of a hold-out sample. Following is a summary of the use of hold-out samples for the studies by decade :

Hold-Out Sample Tested

Hold-Out Sample Not Tested

\begin{tabular}{lcc}
\hline 1970 's & 1 & 0 \\
1980 's & 3 & 4 \\
1990 's & 8 & 8 \\
2000 's & 1 & 2 \\
Overall & $\mathbf{1 3}$ & $\mathbf{1 4}$ \\
\hline
\end{tabular}

\section{ANALYSIS OF RESULTS}

\section{Model Accuracy}

Studies usually report classification accuracies separately for non-going concern firms (those which are not likely to survive) and going concern firms. In addition, the bankruptcy prediction literature refers to Type I and Type II errors, which are applicable to going concern prediction models. Type I errors are the misclassification of bankrupt (non-going concern) firms as non-bankrupt (going concerns). Type II errors are the reverse - non-bankrupt (going concern) firms misclassified as bankrupt (non-going concern) firms. Type I errors are generally considered more costly than Type II errors for several reasons including loss of business (audit clients), damage to a firm's reputation, and potential lawsuits/court costs (see for example Koh [1987]). Therefore, the predictive accuracies discussed here refer to the accuracies obtained for non-going concern firms, unless the results were not presented separately for nongoing concern and going concern firms. Where the results were not separately presented, the overall predictive accuracies are discussed.

The predictive abilities of the models vary across time and method. Considering each decade, the ranges of the models' predictive abilities are:

\begin{tabular}{lcll} 
& $\begin{array}{l}\text { Lowest } \\
\text { Accuracy }\end{array}$ & $\begin{array}{l}\text { Highest } \\
\text { Accuracy }\end{array}$ & $\begin{array}{l}\text { Method(S) Used To Obtain } \\
\text { Highest Accuracy }\end{array}$ \\
\hline 1970 's & $93 \%$ & $93 \%$ & MDA [McKee, 1976] \\
1980 's & $78 \%$ & $100 \%$ & logit analysis [Menon and Schwartz, 1987] \\
1990 's & $32 \%$ & $100 \%$ & neural network [Koh and Tan, 1999] \\
2000 's & $77 \%$ & $92 \%$ & Hybrid of MDA and an expert system [Lenard et al., 2001] \\
\hline
\end{tabular}

Over time, the range of model accuracies has remained consistent, although only two models ([Menon and Schwartz, 1987]; [Koh and Tan, 1999]) have achieved 100\% accuracy. Disregarding Hansen et al.'s [1992] model, which yielded only $32 \%$ accuracy, all of the models which reported classification accuracies had $60 \%$ or higher accuracy. These results do not suggest that newer models are more promising than older models. 

models are:

Considering the primary methods used in model development, the ranges of predictive abilities achieved by

\begin{tabular}{lccl} 
& $\begin{array}{c}\text { Lowest } \\
\text { Accuracy }\end{array}$ & $\begin{array}{c}\text { Highest } \\
\text { Accuracy }\end{array}$ & $\begin{array}{l}\text { Studies which obtained } \\
\text { Highest Accuracy }\end{array}$ \\
\hline MDA & $78 \%$ & $94 \%$ & Barnes and Huan [1993] \\
Logit analysis & $60 \%$ & $100 \%$ & Menon and Schwartz [1987] \\
Probit analysis & $83 \%$ & $86 \%$ & Koh [1987] \\
Neural network & $79 \%$ & $100 \%$ & Koh and Tan [1999] \\
\hline
\end{tabular}

Logit analysis and neural network models have provided the highest success rates, with models that achieved $100 \%$ classification accuracy. This is not surprising because, as discussed earlier in the paper, logit analysis and neural networks have been used most frequently to develop models. With the best accuracy range (79\% to $100 \%$ ) of the various methods, neural networks appear to be the most promising models for going concern prediction.

\section{Prediction Timeframe}

It is also important to consider how far ahead the model is able to accurately predict a firm's going concern status. Clearly, a model that is able to accurately predict a firm's going concern status earlier becomes more valuable. Many of the studies did not publish the models' predictive abilities broken down by the length of time prior to the firm receiving a going concern opinion. However, three of the studies ([McKee, 1976]; [Udo, 1993]; [Lacher et al., 1995]) did report results in this manner. For example, Lacher et al.'s [1995] model predicted non-going concerns with 92\% accuracy one to two years prior. However, McKee's [1976] model could predict that a firm would not be a going concern with $93 \%$ accuracy three years prior.

\section{Validation Method}

The predictive ability of a model can also be impacted by whether the results are from tests of an estimation sample or a hold-out sample. Results from an estimation sample tend to be higher because the model is calculated based on that sample. As mentioned previously, a better indication of a model's validity is obtained by testing a holdout sample. Both of the studies ([Menon and Schwartz, 1987]; [Koh and Tan, 1999]) that obtained 100\% classification accuracy reported these results based on tests of a hold-out sample.

\section{Number Of Factors (Variables)}

Another issue related to the predictive abilities of models is the number of factors considered in the model. The models ([Menon and Schwartz, 1987]; [Koh and Tan, 1999]) that provided 100\% classification accuracy considered seven and six factors, respectively. Yet other models that considered six and seven factors had accuracies ranging from $60 \%$ to $93 \%$. The model [Barnes and Huan, 1993] that considered the most factors (117) had a predictive accuracy of 94\%. However, models ([Chen and Church, 1992]; [Lenard et al., 1995]) that considered only eight factors yielded accuracies of $98 \%$ and $95 \%$, respectively. Therefore, a higher number of factors does not guarantee a higher predictive ability.

\section{CONCLUSIONS}

Asare [1990] previously presented a comprehensive summary of going concern prediction research and model development. This paper contributes to the literature by updating Asare's efforts and by outlining the research concerning the development of going concern prediction models. This paper also makes these contributions: (1) summarizes statistics on model attributes, such as the number of factors and method used; (2) discusses the factors used most frequently in studies; (3) breaks down the predictive accuracies of the models by decade; (4) compares model accuracies based on the method used for model development; (5) identifies studies that used a hold-out sample for validation; and (6) provides a summary of studies involving non-U.S. firms. 
Asare [1990] made several suggestions for future research. His suggestions for future research included examination of:

- the impact of the going concern report on suppliers of debt capital, investment and production decisionmaking, regulatory agencies, and labor/management relations and contract negotiations;

- the argument that going concern assessment is useful because auditors can use qualification as leverage to force disclosures that may not be reported otherwise and other aspects of negotiations between auditors and clients regarding the going concern report;

- $\quad$ whether the going concern report is a self-fulfilling prophecy; and

- $\quad$ whether the going concern report provides protection to auditors from lawsuits.

Table 1

Models For Assessing Going Concern

\begin{tabular}{|c|c|c|c|c|c|c|}
\hline Model & $\begin{array}{c}\begin{array}{c}\text { Purpose of } \\
\text { Model }^{3}\end{array} \\
\end{array}$ & Type of Model & \multicolumn{4}{|c|}{ Model Accuracy } \\
\hline $\begin{array}{l}\text { McKee } \\
(1976)\end{array}$ & $\begin{array}{l}\text { Going concern } \\
\text { prediction }\end{array}$ & $\begin{array}{l}\text { Multivariate } \\
\text { discriminant } \\
\text { analysis } \\
\text { (7 factors) }\end{array}$ & \multicolumn{3}{|c|}{$\begin{array}{lc}\text { Year before failurel } & 2 \\
\text { Model accuracy (using 1 year of data) for hold-out sample: } & 87 \% \\
\text { Non-going concern firms } 87 \% & 60 \% \\
\text { Going concern firms } 47 \% & \\
& \\
\text { Model accuracy (using 3 years of data) for hold-out sample: } & \\
\text { Non-going concern firms - 89\% } & \\
\text { Going concern firms - 56\% } & \end{array}$} & $\begin{array}{r}3 \\
93 \% \\
60 \%\end{array}$ \\
\hline $\begin{array}{l}\text { Kida } \\
(1980)\end{array}$ & $\begin{array}{l}\text { Going concern } \\
\text { prediction } \\
\text { Manufacturing } \\
\text { firms }\end{array}$ & $\begin{array}{l}\text { Multivariate } \\
\text { discriminant } \\
\text { analysis } \\
\text { (5 factors) } \\
\text { Auditor judgment } \\
\text { (5 factors) }\end{array}$ & \multicolumn{4}{|c|}{$\begin{array}{l}\text { Model accuracy: } \\
\quad \text { Problem firms }-85 \% \\
\quad \text { Non-problem firms }-95 \% \\
\text { Auditor judgment accuracy }-83 \%\end{array}$} \\
\hline $\begin{array}{l}\text { Williams } \\
\text { (1982) }\end{array}$ & $\begin{array}{l}\text { Going concern } \\
\text { prediction } \\
\text { Manufacturing } \\
\text { firms }\end{array}$ & $\begin{array}{l}\text { Judgmental } \\
\text { ( } 25 \text { factors) }\end{array}$ & \multicolumn{4}{|c|}{$\begin{array}{l}\text { Model accuracy: } \\
\text { National level firm participants }-86 \% \\
\text { Local level firm participants }-79 \% \\
\text { Regional and local level firm participants }-73 \%\end{array}$} \\
\hline $\begin{array}{l}\text { Mutchler } \\
(1985)\end{array}$ & $\begin{array}{l}\text { Going concern } \\
\text { prediction } \\
\text { Manufacturing } \\
\text { firms }\end{array}$ & $\begin{array}{l}\text { Multivariate } \\
\text { discriminant } \\
\text { analysis } \\
\text { (9 factors) }\end{array}$ & $\begin{array}{l}\text { } \\
\text { Ratios Only } \\
\text { Ratios and Good/ } \\
\text { Bad News Variable } \\
\text { Ratios and Improvement } \\
\text { Over Prior Year Variable } \\
\text { Ratios and Prior Year Goin }\end{array}$ & $\begin{array}{l}\text { with two or } \\
\text { onsecutive } \\
\text { concern opinions } \\
\text { accuracy: } \\
82.8 \% \\
80.7 \% \\
82.6 \% \\
\text { ern Variable }-89\end{array}$ & $\begin{array}{l}\begin{array}{r}\text { Firms } w \\
\text { going co } \\
\text { s opinion }\end{array} \\
83.0 \% \\
80.2 \% \\
81.8 \% \\
9.9 \%\end{array}$ & $\begin{array}{l}\text { nitial } \\
\text { rn }\end{array}$ \\
\hline
\end{tabular}

3 Unless otherwise specified, models are assumed to have been developed for application to medium and large manufacturing and retail firms (SIC codes 2000 to 3999 and 5000 to 5999). This "general" application does not include small businesses, financial/insurance/real estate firms (SIC codes 6000 and above), or transportation firms and utilities (SIC codes 4000-4999). 
Table 1: (Continued)

Models For Assessing Going Concern

\begin{tabular}{|c|c|c|c|}
\hline Model & $\begin{array}{c}\text { Purpose of } \\
\text { Model }\end{array}$ & Type of Model & Model Accuracy \\
\hline $\begin{array}{l}\text { Dopuch, } \\
\text { Holthausen } \\
\text { and Leftwich } \\
\text { (1987) }\end{array}$ & $\begin{array}{l}\text { Going concern } \\
\text { prediction } \\
\text { Firms with SIC } \\
\text { codes other than } \\
6000-6999\end{array}$ & $\begin{array}{l}\text { Probit analysis } \\
\text { (9 factors) }\end{array}$ & $\begin{array}{l}\text { Hold-out sample's estimated probability of: Going concern opinion in } \\
\text { year of qualification - mean } .305 \text { ( } 12 \% \text { of firms actually received going } \\
\text { concern opinion) } \\
\text { Type I error in year of qualification (misclassification cost ratio } 20: 1 \text { ) - } \\
.167\end{array}$ \\
\hline $\begin{array}{l}\text { Koh } \\
\text { (1987) }\end{array}$ & $\begin{array}{l}\text { Going concern } \\
\text { prediction }\end{array}$ & $\begin{array}{l}\text { Probit analysis } \\
\text { (6 factors) }\end{array}$ & $\begin{array}{l}\text { Model accuracy: } \\
\text { Bankrupt firms }-85.45 \% \\
\text { Non-bankrupt firms }-100 \% \\
\text { Auditor issued going concern opinion: } \\
\text { Bankrupt firms }-54.37 \% \\
\text { Non-bankrupt firms }-0 \%\end{array}$ \\
\hline $\begin{array}{l}\text { Menon and } \\
\text { Schwartz } \\
(1987)\end{array}$ & $\begin{array}{l}\text { Going concern } \\
\text { prediction } \\
\text { Firms with SIC } \\
\text { codes other than } \\
6000-6999\end{array}$ & $\begin{array}{l}\text { Logit analysis } \\
\text { (7 factors) }\end{array}$ & $\begin{array}{l}\text { Model accuracy for hold-out sample }(p<.1 \text { or } \geq .9) \text { : } \\
\text { Bankrupt firms }-100 \% \\
\text { Non-bankrupt firms }-93.8 \% \text { (Note: } 10 \text { firms not receiving } \\
\text { qualification were also assigned } .5 \text { chance or higher of receiving } \\
\text { qualification by the model) } \\
\text { Auditor issued going concern opinion }-32.6 \% \\
\text { Auditor disclaimed issuing an opinion due to going concern reason - } \\
9.0 \%\end{array}$ \\
\hline $\begin{array}{l}\text { Harris } \\
\text { (1989) }\end{array}$ & $\begin{array}{l}\text { Going concern } \\
\text { prediction }\end{array}$ & $\begin{array}{l}\text { Recursive } \\
\text { partitioning } \\
\text { algorithm } \\
\text { (13 factors) } \\
\text { Multivariate } \\
\text { discriminant } \\
\text { analysis } \\
\text { (13 factors) } \\
\text { Logit analysis } \\
\text { (13 factors) }\end{array}$ & $\begin{array}{l}\text { Model accuracy for hold-out sample: } \\
\text { Bankrupt firms }-77 \% \\
\text { Non-bankrupt firms }-86 \% \\
\text { Bankrupt firms }-78 \% \\
\text { Non-bankrupt firms }-92 \% \\
\text { Bankrupt firms }-72 \% \\
\text { Non-bankrupt firms }-84 \%\end{array}$ \\
\hline
\end{tabular}


Table 1: (Continued)

Models For Assessing Going Concern

\begin{tabular}{|c|c|c|c|}
\hline Model & $\begin{array}{l}\text { Purpose of } \\
\text { Model }\end{array}$ & Type of Model & Model Accuracy \\
\hline $\begin{array}{l}\text { Bell and } \\
\text { Tabor } \\
(1991)\end{array}$ & $\begin{array}{l}\text { Going concern } \\
\text { prediction }\end{array}$ & $\begin{array}{l}\text { Logit analysis } \\
\text { (6 factors) }\end{array}$ & $\begin{array}{l}\text { Model accuracy }(P \leq .5) \text { for hold-out sample: } \\
\text { Qualified firms }-60.2 \% \\
\text { Unqualified firms }-99.2 \%\end{array}$ \\
\hline $\begin{array}{l}\text { Koh and } \\
\text { Brown } \\
(1991)\end{array}$ & $\begin{array}{l}\text { Going concern } \\
\text { prediction }\end{array}$ & $\begin{array}{l}\text { Probit analysis } \\
\text { (7 factors) }\end{array}$ & $\begin{array}{l}\text { Model accuracy: } \\
\text { Bankrupt firms }-82.5 \% \\
\text { Non-bankrupt firms - } 100 \% \\
\\
\text { Auditor issued going concern opinion: } \\
\text { Bankrupt firms - } 40 \% \\
\text { Non-bankrupt firms }-2.5 \%\end{array}$ \\
\hline $\begin{array}{l}\text { McKeown, } \\
\text { Mutchler and } \\
\text { Hopwood } \\
\text { (1991) }\end{array}$ & $\begin{array}{l}\text { Going concern } \\
\text { prediction }\end{array}$ & $\begin{array}{l}\text { Logit analysis } \\
\text { (9 factors) }\end{array}$ & $\begin{array}{l}\text { Findings Related to Stressed Companies: } \\
\text { Bankrupt group - smaller size, bigger losses, more debt, lower } \\
\text { current ratio, higher incidence of fraud reported after audit report } \\
\text { date } \\
\text { Bankrupt, qualified group - higher likelihood of bankruptcy, } \\
\text { smaller size, longer length of time between year end and audit } \\
\text { report date } \\
\text { Auditor issued going concern opinion to bankrupt company - 46\% } \\
\text { Auditor issued going concern opinion to non-bankrupt company - } \\
5 \% \\
\text { Findings Related to Non-Stressed Companies: } \\
\text { Bankrupt group - lower earnings, higher debt, smaller size Non- } \\
\text { bankrupt, non-qualified group - highest incidence of fraud } \\
\text { reported before audit report date } \\
\text { Bankrupt, non-qualified group - highest incidence of fraud reported } \\
\text { after audit report date } \\
\text { Auditor did not issue going concern opinion to any non-stressed } \\
\text { sample companies }\end{array}$ \\
\hline
\end{tabular}


Table 1: (Continued)

Models For Assessing Going Concern

\begin{tabular}{|c|c|c|c|}
\hline Model & $\begin{array}{c}\text { Purpose of } \\
\text { Model }\end{array}$ & Type of Model & Model Accuracy \\
\hline $\begin{array}{l}\text { Chen and } \\
\text { Church } \\
(1992)\end{array}$ & $\begin{array}{l}\text { Going concern } \\
\text { prediction }\end{array}$ & $\begin{array}{l}\text { Logit analysis } \\
\text { (8 factors) }\end{array}$ & $\begin{array}{l}\text { Model accuracy: } \\
\text { Financial Variables Only Model (misclassifications cost ratio 50:1) } \\
\text { Non-going concern firms }-96.8 \% \\
\text { Going concern firms }-44.9 \% \\
\frac{\text { Default Status Only Model }}{\text { Non-going concern firms }-77.2 \%} \\
\text { Going concern firms - } 99.2 \% \\
\text { Financial Variables and Default Status Model (misclassifications cost } \\
\text { ratio 50:1) } \\
\text { Non-going concern firms }-97.6 \% \\
\text { Going concern firms }-83.5 \%\end{array}$ \\
\hline $\begin{array}{l}\text { Hansen, } \\
\text { McDonald } \\
\text { and Stice } \\
(1992)\end{array}$ & $\begin{array}{l}\text { Going concern } \\
\text { prediction } \\
\text { Manufacturing } \\
\text { firms }\end{array}$ & $\begin{array}{l}\text { Generalized } \\
\text { qualitative } \\
\text { response models } \\
\text { (12 factors) } \\
\text { Machine-learning } \\
\text { models } \\
\text { (12 factors) }\end{array}$ & $\begin{array}{l}\text { Model accuracy (average) for hold-out sample: } \\
\text { Exponential generalized beta of the second kind (EGB2) - 32.2\% } \\
\text { Eburr } 12-32.1 \% \\
\text { Eburr } 3-32.0 \% \\
\text { Logit analysis }-32.0 \% \\
\text { Probit analysis }-31.7 \% \\
\text { Neural network }-31.2 \% \\
\text { Inductive Dichotomizer } 3 \text { (ID3) }-30.1 \%\end{array}$ \\
\hline $\begin{array}{l}\text { Barnes and } \\
\text { Huan } \\
(1993)\end{array}$ & $\begin{array}{l}\text { Going concern } \\
\text { prediction } \\
\text { United Kingdom } \\
\text { firms }\end{array}$ & $\begin{array}{l}\text { Multivariate } \\
\text { discriminant } \\
\text { analysis } \\
\text { (11 of } 117 \text { factors) } \\
\text { Auditor judgment } \\
\text { (117 factors) }\end{array}$ & $\begin{array}{l}\text { Model accuracy: } \\
\text { Non-going concern firms }-94 \% \\
\text { Going concern firms }-68 \% \\
76.8 \% \text { of the auditors would have issued a going concern opinion }\end{array}$ \\
\hline
\end{tabular}


Table 1: (Continued)

Models For Assessing Going Concern

\begin{tabular}{|c|c|c|c|c|c|}
\hline Model & $\begin{array}{c}\text { Purpose of } \\
\text { Model }\end{array}$ & Type of Model & \multicolumn{3}{|c|}{ Model Accuracy } \\
\hline $\begin{array}{l}\text { Biggs, } \\
\text { Selfridge and } \\
\text { Krupka } \\
(1993)\end{array}$ & $\begin{array}{l}\text { Going concern } \\
\text { prediction }\end{array}$ & $\begin{array}{l}\text { Computational } \\
\text { model } \\
(9 \text { factors })\end{array}$ & $\begin{array}{l}\text { Study considered a shipping company } \\
\text { Auditors evaluated Merrytime to asse } \\
\text { Computational model judgment of go } \\
\text { judgment made by auditors for all five }\end{array}$ & $\begin{array}{l}\text { called } \\
\text { going } \\
\text { g conc } \\
\text { rears o }\end{array}$ & $\begin{array}{l}\text { me') } \\
\text { status } \\
\text { s matched }\end{array}$ \\
\hline $\begin{array}{l}\text { Udo } \\
(1993)\end{array}$ & $\begin{array}{l}\text { Going concern } \\
\text { prediction }\end{array}$ & $\begin{array}{l}\text { Neural network } \\
\text { (16 factors) } \\
\text { Multiple } \\
\text { regression } \\
\text { (6 of } 16 \text { factors) }\end{array}$ & $\begin{array}{l}\text { Year before failure } \\
\text { Model accuracy for hold-out sample: } \\
\text { Failed firms } \\
\text { Non-failed firms } \\
\text { Failed firms } \\
\text { Non-failed firms }\end{array}$ & $\begin{array}{l}1 \\
86 \% \\
90 \% \\
76 \% \\
80 \% \\
\end{array}$ & $\begin{array}{l}2 \\
84 \% \\
82 \% \\
74 \% \\
72 \%\end{array}$ \\
\hline $\begin{array}{l}\text { Cormier, } \\
\text { Magnan and } \\
\text { Morard } \\
(1995)\end{array}$ & $\begin{array}{l}\text { Going concern } \\
\text { prediction } \\
\text { Canadian firms }\end{array}$ & $\begin{array}{l}\text { Logit analysis } \\
\text { (16 factors) } \\
\text { Linear } \\
\text { discriminant } \\
\text { analysis } \\
\text { (16 factors) } \\
\text { Recursive } \\
\text { partitioning } \\
\text { (16 factors) }\end{array}$ & $\begin{array}{l}\text { Model accuracy: } \\
\text { Failing firms }-76.1 \% \\
\text { Non-failing firms }-76.8 \% \\
\text { Failing firms }-81.9 \% \\
\text { Non-failing firms }-70.5 \% \\
\\
\text { Failing firms }-70.3 \% \\
\text { Non-failing firms }-80.4 \%\end{array}$ & & \\
\hline $\begin{array}{l}\text { Klersey and } \\
\text { Dugan } \\
(1995)\end{array}$ & $\begin{array}{l}\text { Going concern } \\
\text { prediction } \\
\text { Manufacturing } \\
\text { firms }\end{array}$ & $\begin{array}{l}\text { Neural network } \\
\text { (7 factors) }\end{array}$ & $\begin{array}{l}\text { Model accuracy: } \\
\text { Non-going concern firms }-79.4 \% \\
\text { Going concern firms }-67.6 \%\end{array}$ & & \\
\hline
\end{tabular}


Table 1: (Continued)

Models For Assessing Going Concern

\begin{tabular}{|c|c|c|c|c|c|c|c|}
\hline Model & $\begin{array}{c}\text { Purpose of } \\
\text { Model }\end{array}$ & Type of Model & \multicolumn{5}{|c|}{ Model Accuracy } \\
\hline $\begin{array}{l}\text { Lacher, } \\
\text { Coats, } \\
\text { Sharma and } \\
\text { Fant } \\
(1995)\end{array}$ & $\begin{array}{l}\text { Going concern } \\
\text { prediction }\end{array}$ & $\begin{array}{l}\text { Neural network } \\
\text { (5 factors) }\end{array}$ & $\begin{array}{l}\text { Year before going concern } \\
\text { Model accuracy for hold-ou } \\
\text { Non-going concern firms } \\
\text { Going concern firms }\end{array}$ & $\begin{array}{l}\text { ort } \\
\text { sample: } \\
\\
0 \\
89.4 \% \\
97.9 \%\end{array}$ & $\begin{array}{c} \\
1 \\
91.5 \% \\
97.9 \%\end{array}$ & $\begin{array}{c}2 \\
91.5 \% \\
87.2 \%\end{array}$ & $\begin{array}{c} \\
3 \\
89.4 \% \\
78.7 \%\end{array}$ \\
\hline $\begin{array}{l}\text { Lenard, } \\
\text { Alam and } \\
\text { Madey } \\
(1995)\end{array}$ & $\begin{array}{l}\text { Going concern } \\
\text { prediction }\end{array}$ & $\begin{array}{l}\text { Logit analysis } \\
\text { (8 factors) } \\
\text { N-NET neural } \\
\text { network } \\
\text { (8 factors) } \\
\text { GRGx neural } \\
\text { network } \\
\text { (8 factors) }\end{array}$ & $\begin{array}{l}\text { Model accuracy: } \\
\text { Non-going concern firms } \\
\text { Going concern firms } \\
\text { Non-going concern firms } \\
\text { Going concern firms } \\
\text { Non-going concern firms } \\
\text { Going concern firms }\end{array}$ & $\begin{array}{r}\text { Mode } \\
\text { (8 fact } \\
66.7 \\
100 \\
\\
77.5 \\
90.0 \\
\\
87.5 \\
90.0\end{array}$ & & $\begin{array}{r}\text { Model } \\
\text { (4 of } 8 \mathrm{fa} \\
52.5 \% \\
97.5 \% \\
90.0 \% \\
92.5 \% \\
\\
95.0 \% \\
95.0 \%\end{array}$ & ors) \\
\hline $\begin{array}{l}\text { Raghunanda } \\
\mathrm{n} \text { and Rama } \\
(1995)\end{array}$ & $\begin{array}{l}\text { Going concern } \\
\text { prediction }\end{array}$ & $\begin{array}{l}\text { Logit analysis } \\
\text { (9 factors) }\end{array}$ & $\begin{array}{l}\text { Model accuracy: } \\
\text { Excluding 'time' factors } \\
\text { Including time between a } \\
\text { factors) - } 90 \% \\
\text { Also including time betw } \\
92 \% \\
\text { Auditor issued going concer } \\
\text { Pre-SAS No. } 59 \text { sample - } \\
\text { Post-SAS No. } 59 \text { sample }\end{array}$ & $\begin{array}{l}\text { of } 9 \text { fact } \\
\text { it report } \\
\text { n year-er } \\
\\
\text { opinion: } \\
2 \% \\
35 \%\end{array}$ & $\begin{array}{l}\text { includec } \\
\text { e/bankrt } \\
\text { audit rep }\end{array}$ & $\begin{array}{l}-87 \% \\
\text { tcy filing } \\
\text { t date }(9 \mathrm{f}\end{array}$ & of 9 \\
\hline $\begin{array}{l}\text { Mutchler, } \\
\text { Hopwood } \\
\text { and } \\
\text { McKeown } \\
(1997)\end{array}$ & $\begin{array}{l}\text { Going concern } \\
\text { prediction }\end{array}$ & $\begin{array}{l}\text { Logit analysis } \\
\text { (13 factors) }\end{array}$ & $\begin{array}{l}\text { Probability of bankruptcy, } \\
\text { variables add significant ex } \\
\text { Larger client = lower proba } \\
\text { Client with debt default }=\mathrm{h} \\
\text { Client with debt default that } \\
\text { going concern opinion than } \\
\text { Mild news items before/afte }\end{array}$ & $\begin{array}{l}\text { dit-repor } \\
\text { atory p } \\
\text { lity of go } \\
\text { her probs } \\
\text { as been } \\
\text { ient with } \\
\text { udit repc }\end{array}$ & $\begin{array}{l}\mathrm{g} \text {, and } \mathrm{bc} \\
\text { er in ant } \\
\text { concerr } \\
\text { lity of go } \\
\text { ed = hig } \\
\text { debt de } \\
\text { date }=\mathrm{n}\end{array}$ & $\begin{array}{l}\text { kruptcy la } \\
\text { ipated dire } \\
\text { pinion } \\
\text { g concern } \\
\mathrm{r} \text { probabil } \\
\text { ult } \\
\text { significan }\end{array}$ & $\begin{array}{l}\text { tions } \\
\text { pinion } \\
\text { y of }\end{array}$ \\
\hline
\end{tabular}


Table 1: (Continued)

Models For Assessing Going Concern

\begin{tabular}{|c|c|c|c|}
\hline Model & $\begin{array}{c}\text { Purpose of } \\
\text { Model }\end{array}$ & Type of Model & Model Accuracy \\
\hline $\begin{array}{l}\text { Foster, Ward } \\
\text { and } \\
\text { Woodroof } \\
(1998)\end{array}$ & $\begin{array}{l}\text { Going concern } \\
\text { prediction }\end{array}$ & $\begin{array}{l}\text { Logit analysis } \\
\text { (10 factors) }\end{array}$ & $\begin{array}{l}\text { Loan default/accommodation and covenant violations best predict the } \\
\text { bankruptcy of stressed firms at high cost ratios of } 60: 1 \text { or greater } \\
\text { Adding the loan default/accommodation and covenant violations } \\
\text { factors significantly improves model's predictive ability at cost ratios } \\
\text { from } 50: 1 \text { to } 80: 1 \\
\text { Naïve assumption of debt default is better predictor than statistical } \\
\text { model including debt default factors } \\
\text { Going concern opinion factor does not significantly improve model's } \\
\text { predictive ability } \\
\text { Auditor issued going concern opinion: } \\
\text { Bankrupt firms - } 41.5 \%\end{array}$ \\
\hline $\begin{array}{l}\text { Koh and Tan } \\
\text { (1999) }\end{array}$ & $\begin{array}{l}\text { Going concern } \\
\text { prediction }\end{array}$ & $\begin{array}{l}\text { Neural network } \\
\text { (6 factors) }\end{array}$ & $\begin{array}{l}\text { Model accuracy for hold-out sample: } \\
\text { Non-going concern firms - } 100 \% \\
\text { Going concern firms - } 100 \% \\
\text { Auditor issued going concern opinion: } \\
\quad \text { Non-going concern firms }-54.37 \% \\
\text { Going concern firms }-0 \%\end{array}$ \\
\hline $\begin{array}{l}\text { Casterella, } \\
\text { Lewis and } \\
\text { Walker } \\
(2000)\end{array}$ & $\begin{array}{l}\text { Going concern } \\
\text { prediction } \\
\text { Firms with SIC } \\
\text { codes below } \\
6000\end{array}$ & $\begin{array}{l}\text { Logit analysis } \\
\text { (12 factors) }\end{array}$ & $\begin{array}{l}\text { Model is found to be significant }(p=.001) \\
\text { Variables found to be significant are: Altman Z-score, Debt default, } \\
\text { Length of auditor-client relationship, Time between audit report } \\
\text { date/bankruptcy filing, Time between year-end/audit report date } \\
\text { Auditor issued going concern opinion to: } \\
\text { Firms with a probability of bankruptcy }>.5-100 \% \\
\text { Firms not appearing financially distressed }-31 \%\end{array}$ \\
\hline
\end{tabular}


Table 1: (Continued)

Models For Assessing Going Concern

\begin{tabular}{|c|c|c|c|}
\hline Model & $\begin{array}{c}\text { Purpose of } \\
\text { Model }\end{array}$ & Type of Model & Model Accuracy \\
\hline $\begin{array}{l}\text { Lenard, } \\
\text { Alam, Booth } \\
\text { and Madey } \\
(2001)\end{array}$ & $\begin{array}{l}\text { Going concern } \\
\text { prediction }\end{array}$ & $\begin{array}{l}\text { Hybrid system - } \\
\text { Multivariate } \\
\text { discriminant } \\
\text { analysis for } \\
\text { bankruptcy } \\
\text { prediction and } \\
\text { expert system } \\
\text { (11 factors) }\end{array}$ & $\begin{array}{l}\text { Model accuracy with equally weighted risks for hold-out sample: } \\
\text { 1990 data } \\
\text { Modified audit report for bankrupt firms }-80.8 \% \\
\text { Standard audit report for non-bankrupt firms }-92.3 \% \\
\text { 1994-1998 data } \\
\text { Modified audit report for bankrupt firms }-76.9 \% \\
\text { Standard audit report for non-bankrupt firms }-100 \% \\
\text { Model accuracy with 'high operating risk' more heavily weighted for } \\
\text { hold-out sample: } \\
\text { 1990 data } \\
\text { Modified audit report for bankrupt firms }-92.3 \% \\
\text { Standard audit report for non-bankrupt firms - 100\% } \\
\text { 1994-1998 data } \\
\text { Modified audit report for bankrupt firms }-80.8 \% \\
\text { Standard audit report for non-bankrupt firms - } 100 \%\end{array}$ \\
\hline $\begin{array}{l}\text { Gaeremynck } \\
\text { and } \\
\text { Willekens } \\
(2003)\end{array}$ & $\begin{array}{l}\text { Going concern } \\
\text { prediction } \\
\text { Belgian private } \\
\text { firms }\end{array}$ & $\begin{array}{l}\text { Logit analysis } \\
\text { (8 factors) }\end{array}$ & Model accuracy $-77.2 \%$ \\
\hline
\end{tabular}

Table 2:

Factors Included In Five Or More Models ${ }^{4}$

\begin{tabular}{|l|c|}
\hline Factor/Consideration & Number of Models that Include \\
\hline Current ratio & 14 \\
\hline Net income / Total assets & 11 \\
\hline Retained earnings / Total assets & 10 \\
\hline Quick ratio & 9 \\
\hline Cash / Total assets & 8 \\
\hline Total liabilities / Total assets & 8 \\
\hline Cash flow from operations / Total liabilities & 7 \\
\hline Current assets / Total assets & 7 \\
\hline Debt default & 6 \\
\hline Current ratio (one year change) & 5 \\
\hline Long-term debt / Total assets & 5 \\
\hline Net income before tax / Net sales & 5 \\
\hline Sales (log or natural log) & 5 \\
\hline Sales / Total assets & \\
\hline Total debt / Total assets & 5 \\
\hline Working capital / Total assets & 5 \\
\hline
\end{tabular}

\footnotetext{
${ }^{4}$ The complete list of factors and considerations included in each model and a complete list of how many models each of the 237 factors are utilized in are available upon request from the authors.
} 


\section{ANNOTATED BIBLIOGRAPHY}

1. Barnes, P. and H. Huan. 1993. The auditor's going concern decision: Some UK evidence concerning independence and competence. Journal of Business Finance \& Accounting 20(2): 213-228.

Barnes and Huan presented a case study to UK audit partners who were asked to judge whether or not they would issue a going concern opinion for the company in the case. Included in the case were over ninety indications of going concern difficulties and over twenty items of mitigating evidence. The participants were also asked to evaluate the usefulness of these items in making the going concern assessment. The responses provided by the audit partners were used to develop a discriminant analysis model (to determine whether or not a going concern opinion should be issued) which yielded an overall accuracy of $87.96 \%$.

2. Bell, T. and R. Tabor. 1991. Empirical analysis of audit uncertainty qualifications. Journal of Accounting Research 29(2): 350-370.

Bell and Tabor developed a going concern prediction model to assist auditors in the decision process for whether or not to modify the audit report for going concern uncertainties. The authors first performed univariate tests and then developed a multivariate model using logit analysis. Hold-out tests were performed on a sample of 108 firms that received going concern qualified opinions and 995 firms that received opinions not qualified for going concern. Hold-out tests yielded classification accuracies of $99.2 \%$ of the unqualified firms and $60.2 \%$ of the qualified firms.

3. Biggs, S., M. Selfridge and G. Krupka. 1993. A computational model of auditor knowledge and reasoning processes in the going-concern judgment. Auditing: A Journal of Practice \& Theory 12: 82+.

The authors developed a computational model to simulate the going concern evaluation process of auditors. The authors interviewed a partner and a manager of a large accounting firm to collect information on how they make a going concern judgment. Information from the interview was used to program the computational model. The model judged the going concern assessment the same as the auditors did for each of five years.

4. Casterella, J., B. Lewis and P. Walker. 2000. Modeling the audit opinions issued to bankrupt companies: A two-stage empirical analysis. Decision Sciences 31(2): 507-530.

The authors developed a going concern opinion prediction model using logit analysis. The result of this model was then used in a second model to predict bankruptcy resolution. The model's classification accuracy was not provided, though the authors stated that the model was found to be "highly significant". The results of the model were compared with Zmijewski's [1984] bankruptcy prediction model and the actual auditor opinion. The authors concluded that the auditors' decisions were more accurate than the bankruptcy prediction model.

5. Chen, K. and B. Church. 1992. Default on debt obligations and the issuance of going-concern opinions. Auditing: A Journal of Practice \& Theory 11(2): 30-49.

Chen and Church investigated the usefulness of default status in a going concern opinion prediction model using logit analysis. The authors developed three models - one using seven financial factors, one using just default status, and one combining the financial factors and default status. Validation tests were performed using a jackknife approach and using a subset of the original sample. Results are provided for six levels of misclassification costs ratios. The authors found that, overall, the combined model most accurately predicted the opinion.

6. Cormier, D., M. Magnan and B. Morard. 1995. The auditor's consideration of the going concern assumption: A diagnostic model. Journal of Accounting, Auditing \& Finance 10(2): 201-222.

The authors developed three models for predicting a going concern opinion for Canadian firms - a linear discriminant analysis model, a logit model, and a recursive partitioning model. Several variables were found to be significant including increases in adjusted returns, increases in debt maturities, increases in payments to shareholders, expansion, and investments in new ventures. Overall classification accuracies do not differ greatly between the three 
methods. The logit model had an overall accuracy of $76.4 \%$; the discriminant analysis had $76.8 \%$ accuracy; and the recursive partitioning model had $74.8 \%$ overall accuracy.

7. Dopuch, N., R. Holthausen and R. Leftwich. 1987. Predicting audit qualifications with financial and market variables. The Accounting Review 63(3): 431-453.

Using probit analysis, the authors developed a model to predict whether a firm will receive a qualified opinion. The authors identify four groups of qualifications - going concern, litigation, asset realizing, and multiple. Hold-out tests performed on 57 firms with qualified opinions and 95 firms with clean opinions showed that firms had a .305 mean probability of receiving a going concern opinion. The authors found that the model had the highest predictive accuracy for going concern qualifications.

8. Foster, B., T. Ward and J. Woodruff. 1998. An analysis of the usefulness of debt defaults and going concern opinions in bankruptcy risk assessment. Journal of Accounting, Auditing, and Finance: 351-371.

The authors applied logit analysis to develop a bankruptcy prediction model which included loan defaults/accommodations and violations of loan covenant requirements. The authors stated that the inclusion of these two factors would allow assessment of the incremental value of the going concern opinion beyond earlier publicly available information. Results showed that the inclusion of these two factors improved the accuracy of the bankruptcy prediction model. The authors also analyzed and provided the estimated misclassification costs from a holdout sample. They reported that the two variables best predicted bankruptcy when estimated misclassification costs were high (ratios greater than or equal to 60 to 1 ).

9. Gaeremynck, A. and M. Willekens. 2003. The endogenous relationship between audit-report type and business termination: Evidence on private firms in a non-litigious environment. Accounting and Business Research 33(1): 65-79.

Gaeremynck and Willekens investigated the relationship between the issuance of a going concern opinion and subsequent business failure for Belgian private firms. The authors developed both an audit report type prediction model and a bankruptcy prediction model. Results showed that the audit report type model could predict the likelihood of a non-clean (qualified or adverse) audit opinion due to going concern issues or GAAP violations with $77.2 \%$ accuracy. GAAP violations were included because they could be indicative of the company's likelihood of survival.

10. Hansen, J., J. McDonald and J. Stice. 1992. Artificial intelligence and generalized qualitative-response models: An empirical test on two audit decision-making domains. Decision Sciences 23(3): 708-723.

The authors developed several models for predicting the issuance of a going concern opinion for manufacturing firms. Results were reported and compared for probit, logit, ID3, and neural network models. Though the results were not exceptional for any of the models, the authors concluded that the qualitative-response models (including logit and probit) performed at a level comparable to the machine-learning models (neural network and ID3).

11. Harris, C. 1989. An expert decision support system for auditor going concern evaluation. Ph.D. dissertation, University of Texas-Arlington.

Harris developed an expert system based on a recursive partitioning algorithm for the prediction of whether or not a firm will receive a going concern opinion. She compared the results of the expert system with both logit analysis and multivariate discriminant analysis models. Initial tests yielded accuracy rates as high as $99 \%$. However, hold-out tests' accuracies for bankrupt firms were $72 \%$ using logit analysis, $78 \%$ using multivariate discriminant analysis, and $77 \%$ using recursive portioning algorithm.

12. Kida, T. 1980. An investigation into auditors' continuity and related qualification judgments. Journal of Accounting Research 18(2): 506-523. 
Kida investigated the going concern decision-making process. He surveyed auditors regarding both the going concern judgment and compared the results with a multivariate discriminant analysis opinion prediction model. The model's accuracy ranged from $85 \%$ to $95 \%$; the auditors' judgments were accurate in $83 \%$ of the cases.

13. Klersey, G. and M. Dugan. 1995. Substantial doubt: Using artificial neural networks to evaluate going concern. Article in Advances in Accounting Information Systems Volume 3, S. Sutton (ed.). Greenwich, CT: JAI Press, Inc., 137-159.

Klersey and Dugan developed a neural network for predicting the issuance of a going concern opinion. Results indicated that the model could accurately predict $79.4 \%$ of non-going concern firms (firms issued a going concern opinion) and $67.6 \%$ of going concern firms.

14. Koh, H. 1987. Prediction of going-concern status: A probit model for the auditors. Ph.D. dissertation, Virginia Polytechnic Institute and State University.

Koh used probit analysis to develop a going concern prediction model. The initial sample yielded classification accuracies of $87 \%$ for bankrupt firms and $100 \%$ for non-bankrupt firms. Validation tests using the Lachenbruch method yielded classification accuracies of $85 \%$ and $100 \%$ for bankrupt and non-bankrupt firms, respectively. Koh also investigated and reported on the impact of expected misclassification costs.

15. Koh, H. and R. Brown. 1991. Probit prediction of going and non-going concerns. Managerial Auditing Journal 6(3): 18-23.

Koh and Brown explored the potential of using probit analysis to develop a going concern prediction model that would overcome many of the perceived deficiencies of discriminant analysis models. In-sample and validation tests (using the Lachenbruch method) yielded identical accuracies of $82.5 \%$ for non-going concern firms. The authors compared the accuracy of the model with actual auditors' opinions. A going concern opinion was issued in only $40 \%$ of the cases for bankrupt (non-going concern firms).

16. Koh, H. and S. Tan. 1999. A neural network approach to the prediction of going concern status. Accounting and Business Research 29(3): 211-216.

Koh and Tan expanded Koh's [1987] work and explored the use of neural networks for going concern prediction. Hold-out tests performed on 30 firms yielded 100\% accuracy for both non-going concern and going concern firms. The authors compared this with the number of cases in which the auditor issued a going concern opinion to non-going concern firms (only $54 \%$ of cases).

17. Lacher, R., P. Coats, S. Sharma and L. Fant. 1995. A neural network for classifying the financial health of a firm. European Journal of Operational Research 85(1): 53-65.

The authors presented a neural network model for going concern opinion prediction. The training sample yielded classification accuracies ranging from $95 \%$ in the year the going concern opinion was received to $86 \%$ three years prior. Hold-out tests performed yielded classification accuracy of $89 \%$ in the year the going concern opinion was received and $92 \%$ the year prior.

18. Lenard, M., P. Alam and G. Madey. 1995. The application of neural networks and a qualitative response model to the auditor's going concern uncertainty decision. Decision Sciences 26(2): 209-227.

Lenard, Alam and Madey presented two neural network models and one logit model for bankruptcy prediction. Using a sample of 80 firms (70 firms for training and 10 firms for testing), the authors ran eight iterations and presented the combined results for each model over all the iterations. Results indicated that the GRGx neural network model, yielding $87.5 \%$ accuracy for non-going concern firms, outperformed the N-NET neural network and logit models. 
19. Lenard, M., P. Alam, D. Booth and G. Madey. 2001. Decision-making capabilities of a hybrid system applied to the auditor's going concern assessment. International Journal of Intelligent Systems in Accounting, Finance \& Management 10(1): 1-24.

The authors developed and tested a hybrid system for going concern opinion prediction. The system, which combined quantitative and qualitative factors, utilized multivariate discriminant analysis in conjunction with an expert system. Classification accuracy has high as $92 \%$ was achieved on hold-out tests.

20. McKee, T. 1976. Discriminant prediction of going concern status: A model for auditors. Selected Papers of the AAA Annual Meeting (1976).

McKee developed a multivariate discriminant analysis model for predicting a firm's going concern status. The initial sample of 35 firms yielded classification accuracies for bankrupt firms of $89 \%$ one year prior to failure and $80 \%$ two and three years prior to failure. Hold-out tests yielded classification accuracies for bankrupt firms of $87 \%$ one and two years prior to failure and $93 \%$ three years prior to failure.

21. McKeown, J., J. Mutchler and W. Hopwood. 1991. Towards an explanation of auditor failure to modify the audit opinions of bankrupt companies. Auditing: A Journal of Practice \& Theory 10: 1-13.

The authors investigated and reported several findings with respect to the modification of the audit opinion for going concern. A model for predicting a firm's going concern status was also presented. The usefulness of a hidden fraud variable was tested as well. The authors did not report the classification accuracies of the prediction model. However, the conclusions indicated that the hidden fraud variable was useful for predicting going concern firms but not for predicting non-going concern (failing) firms.

22. Menon, K. and K. Schwartz. 1987. An empirical investigation of audit qualification decisions in the presence of going concern uncertainties. Contemporary Accounting Research 3(2): 302-315.

Menon and Schwartz developed a model for predicting the going concern status of a firm using logistic regression (logit analysis). Two factors were found to be significant - the change in the current ratio and the presence of recurring operating losses. Results indicated that in the cases where the model was most confident ( $p<0.1$ or $p \geq$ 0.9 ), the model yielded $100 \%$ classification accuracy for bankrupt firms. For non-bankrupt companies in this same interval, the model only misclassified one of sixteen firms.

23. Mutchler, J. 1985. A multivariate analysis of the auditor's going-concern opinion decision. Journal of Accounting Research 23(2): 668-682.

Mutchler investigated the going concern decision process and developed a model to predict the outcome. She presented several models - one which considered ratios only, one which considered ratios plus a good news/bad news variable, one which considered ratios and a variable indicating if there was improvement over the prior year, and one which considered ratios plus a variable indicating if a going concern opinion was issued in the prior year. Results indicated that the good news/bad news variable lowered the model's predictive accuracy. The inclusion of a variable indicating if there was improvement over the prior year had little impact on the model's predictive accuracy. The variable indicating if a going concern opinion was issued in the prior year improved the model's accuracy.

24. Mutchler, J, W. Hopwood and J. McKeown. 1997. The influence of contrary information and mitigating factors on audit opinion decisions on bankrupt companies. Journal of Accounting Research 35(2): 295-310.

The authors investigated the impact of contrary information (such as debt default) and mitigating factors (such as financing sources) on audit opinion decisions for firms that were nearing bankruptcy. The going concern opinion prediction model that was developed considered factors such as audit report lag, good news factors, bad news factors, debt default, firm size, and whether the auditor was a Big Six firm. Results indicated that factors with 
significant explanatory power were the probability of bankruptcy, audit report lag, and bankruptcy lag. The authors also reported that debt payment default and covenant violation were significant, but cured default was not significant.

25. Raghunandan, K. and D. Rama. 1995. Audit reports for companies in financial distress: Before and after SAS No. 59. Auditing: A Journal of Practice \& Theory 14(1): 50-63.

Raghunandan and Rama presented a logit model for predicting the going concern opinion of a firm. Results indicated that five factors were significant - the current ratio, leverage, recurring operating losses, the ratio of cash flow to total liabilities, and the time period (pre- or post-SAS No. 59). The model yielded the highest classification accuracy when it included a factor considering the time between year-end and the issuance of the audit report.

26. Udo, G. 1993. Neural network performance on the bankruptcy classification problem. Computers and Industrial Engineering 25(1-4): 377-380.

Udo investigated the use of neural networks for predicting the going concern opinion of a firm. The neural network's results were compared with the predictive accuracy of a multiple regression model. Results indicated that the neural network performed equal to or better than the regression model in all cases for the hold-out sample.

27. Williams, H. 1982. Cue utilization in auditors' going-concern qualification judgments: An empirical analysis. Ph.D. dissertation, University of Georgia.

Williams investigated the relative importance of the qualitative factors presented in SAS No. 34 The Auditor's Considerations when a Question Arises About an Entity's Continued Existence in the going concern decision. A selection of auditor participants were presented with sample cases and asked to judge the going concern status of each firm. Williams found that participants accurately judged the going concern status of firms in $79 \%$ of the cases on average. Further, Williams concluded that qualitative factors are important to the auditor's evaluation of a firm's going concern status.

\section{REFERENCES}

1. American Institute of Certified Public Accountants (AICPA). 1963. Statement on Auditing Procedures No. 33.

2. 1974. Statement on Auditing Standards No. 2 - Reports on Audited Financial Statements.

3. 1981. Statement on Auditing Standards No. 34 - The Auditor's Considerations When a Question Arises About an Entity's Continued Existence.

4. 1988. Statement on Auditing Standards No. 59 - The Auditor's Consideration of an Entity's Ability to Continue as a Going Concern.

5. Asare, S. 1990. The auditor's going-concern decision: A review and implications for future research. Journal of Accounting Literature 9: 39-64.

6. Barnes, P. and H. Huan. 1993. The auditor's going concern decision: Some UK evidence concerning independence and competence. Journal of Business Finance \& Accounting 20(2): 213-228.

7. Bell, T. and R. Tabor. 1991. Empirical analysis of audit uncertainty qualifications. Journal of Accounting Research 29(2): 350-370.

8. Bellovary, J., D. Giacomino and M. Akers. 2005. A review of bankruptcy prediction studies: 1930 to present. Unpublished manuscript.

9. Biggs, S., M. Selfridge and G. Krupka. 1993. A computational model of auditor knowledge and reasoning processes in the going-concern judgment. Auditing: A Journal of Practice \& Theory 12: 82+.

10. Casterella, J., B. Lewis, and P. Walker. 2000. Modeling the audit opinions issued to bankrupt companies: A twostage empirical analysis. Decision Sciences 31(2): 507-530.

11. Chen, K. and B. Church. 1992. Default on debt obligations and the issuance of going-concern opinions. Auditing: A Journal of Practice \& Theory 11(2): 30-49.

12. Commission on Auditors' Responsibilities (Cohen Commission). 1978. Report, Conclusions, and Recommendations. AICPA: New York.

13. Cormier, D., M. Magnan, and B. Morard. 1995. The auditor's consideration of the going concern assumption: A diagnostic model. Journal of Accounting, Auditing \& Finance 10(2): 201-222. 
14. Dimitras, A., S. Zanakis and C. Zopounidis. 1996. A survey of business failures with an emphasis on prediction methods and industrial applications. European Journal of Operational Research 90: 487-513.

15. Dopuch, N., R. Holthausen, and R. Leftwich. 1987. Predicting audit qualifications with financial and market variables. The Accounting Review 63(3): 431-453.

16. Foster, B., T. Ward, and J. Woodruff. 1998. An analysis of the usefulness of debt defaults and going concern opinions in bankruptcy risk assessment. Journal of Accounting, Auditing, and Finance: 351-371.

17. Gaeremynck, A. and M. Willekens. 2003. The endogenous relationship between audit-report type and business termination: Evidence on private firms in a non-litigious environment. Accounting and Business Research 33(1): 65-79.

18. Geiger, M. and K. Raghunandan. 2002. Going-concern opinions in the "new" legal environment. Accounting Horizons 16 (1): 17-26.

19. __ and D. Rama. 2005. Recent changes in the association between bankruptcies and prior audit opinions. Auditing: A Journal of Practice \& Theory 24(1): 21-35.

20. Hansen, J., J. McDonald, and J. Stice. 1992. Artificial intelligence and generalized qualitative-response models: An empirical test on two audit decision-making domains. Decision Sciences 23(3): 708-723.

21. Harris, C. 1989. An expert decision support system for auditor going concern evaluation. Ph.D. dissertation, University of Texas-Arlington.

22. Jones, F. 1987. Current techniques in bankruptcy prediction. Journal of Accounting Literature 6: 131-164.

23. Kida, T. 1980. An investigation into auditors' continuity and related qualification judgments. Journal of Accounting Research 18(2): 506-523.

24. Klersey, G. and M. Dugan. 1995. Substantial doubt: Using artificial neural networks to evaluate going concern. Article in Advances in Accounting Information Systems Volume 3, S. Sutton (ed.). Greenwich, CT: JAI Press, Inc., 137-159.

25. Koh, H. 1987. Prediction of going-concern status: A probit model for the auditors. Ph.D. dissertation, Virginia Polytechnic Institute and State University.

26. $\quad$ and R. Brown. 1991. Probit prediction of going and non-going concerns. Managerial Auditing Journal 6(3): $18-23$.

27. and S. Tan. 1999. A neural network approach to the prediction of going concern status. Accounting and Business Research 29(3): 211-216.

28. Lacher, R., P. Coats, S. Sharma, and L. Fant. 1995. A neural network for classifying the financial health of a firm. European Journal of Operational Research 85(1): 53-65.

29. Lenard, M., P. Alam and G. Madey. 1995. The application of neural networks and a qualitative response model to the auditor's going concern uncertainty decision. Decision Sciences 26(2): 209-227.

30. _ _ _ D. Booth, and _ 2001. Decision-making capabilities of a hybrid system applied to the auditor's going concern assessment. International Journal of Intelligent Systems in Accounting, Finance \& Management 10(1): 1-24.

31. McKee, T. 1976. Discriminant prediction of going concern status: A model for auditors. Selected Papers of the AAA Annual Meeting (1976).

32. McKeown, J., J. Mutchler, and W. Hopwood. 1991. Towards an explanation of auditor failure to modify the audit opinions of bankrupt companies. Auditing: A Journal of Practice \& Theory 10: 1-13.

33. Menon, K. and K. Schwartz. 1987. An empirical investigation of audit qualification decisions in the presence of going concern uncertainties. Contemporary Accounting Research 3(2): 302-315.

34. Mutchler, J. 1983. A multivariate analysis of auditor decision making in the presence of going-concern uncertainties. Ph.D. dissertation, University of Illinois-Urbana-Champaign.

35. 1985. A multivariate analysis of the auditor's going-concern opinion decision. Journal of Accounting Research 23(2): 668-682.

36. Mutchler, J, W. Hopwood, and J. McKeown. 1997. The influence of contrary information and mitigating factors on audit opinion decisions on bankrupt companies. Journal of Accounting Research 35(2): 295-310.

37. Raghunandan, K. and D. Rama. 1995. Audit reports for companies in financial distress: Before and after SAS No. 59. Auditing: A Journal of Practice \& Theory 14(1): 50-63.

38. Sarbanes-Oxley Act (SOX). 2002. Public Law 107-204.

39. Securities and Exchange Commission (SEC). 1962. Accounting Series Release No. 90.

40. Udo, G. 1993. Neural network performance on the bankruptcy classification problem. Computers and Industrial Engineering 25(1-4): 377-380.

41. Williams, H. 1982. Cue utilization in auditors' going-concern qualification judgments: An empirical analysis. Ph.D. dissertation, University of Georgia. 